\title{
Compliance with the 24-h movement guidelines and the relationship with anthropometry in Finnish preschoolers: the DAGIS study
}

Marja H. Leppänen ${ }^{1,2^{*}}$ (D), Carola Ray ${ }^{1,2}$, Heini Wennman ${ }^{3}$, Christina Alexandrou ${ }^{4}$, Katri Sääksjärvi ${ }^{5}$, Leena Koivusilta ${ }^{6}$, Maijaliisa Erkkola ${ }^{2}$ and Eva Roos ${ }^{1,2,7}$

\begin{abstract}
Background: Recent 24-h movement guidelines for the early years established recommendations for physical activity (PA), screen time (ST), and sleep. To date, few studies have focused on compliance with meeting the guidelines and their associations with health outcomes. Thus, we aimed to investigate: 1) compliance with the 24-h movement guidelines, and 2) associations between compliance and anthropometry in Finnish preschoolers.

Methods: We utilized DAGIS survey data that were collected in 2015-2016 ( $N=864)$. PA was assessed $24 \mathrm{~h} /$ day over 7 days using a waist-worn ActiGraph wGT3X-BT accelerometer. ST and sleep were reported by the parents during the same 7 days. Anthropometry was assessed using body mass index (BMl, $\left.\mathrm{kg} / \mathrm{m}^{2}\right)$ and waist circumference (WC, cm). Children were classified as meeting the guidelines if they averaged $\geq 180 \mathrm{~min} /$ day of PA, which consisted of $\geq 60 \mathrm{~min}$ of moderate-to-vigorous intensity; $\leq 60 \mathrm{~min} /$ day of ST; and 10-13 h/day of sleep. In total, 778 children ( $51 \%$ boys, mean age: $4.7 \pm 0.9$ years) were included in the study. The compliance with meeting the $24-h$ movement guidelines was calculated for each behavior separately and in combinations. Adjusted linear regression analyses were applied to examine associations of compliance with BMI and WC.

Results: Children were physically active on average $390( \pm 46.2) \mathrm{min} /$ day and spent $86( \pm 25.5) \mathrm{min} /$ day in moderate-to-vigorous PA. They spent $76( \pm 37.4) \mathrm{min} /$ day on ST and had on average 10:21 $( \pm 0: 33) \mathrm{h}: \mathrm{min} /$ day of sleep. The compliance rate in meeting all three movement guidelines overall was $24 \%$. The highest compliance rate was found for PA (85\%), followed by sleep (76\%) and ST (35\%). Meeting guidelines separately for PA or sleep, or for both, were associated with lower WC (PA: $B=-1.37, p<0.001$; Sleep: $B=-0.72, p=0.009$; PA + Sleep: $B=-1.03, p<$ 0.001). In addition, meeting guidelines for sleep or for both PA and sleep were associated with lower BMI (Sleep: $\mathrm{B}=-0.26, p=0.027 ; \mathrm{PA}+$ Sleep: $\mathrm{B}=-0.30, p=0.007$ ). There were no significant associations found regarding $\mathrm{ST}$.

Conclusions: Meeting recommendations for PA and sleep may have an important role in supporting a healthy weight status in young children. However, there is still a need to improve compliance with the 24-h movement guidelines, especially for ST.
\end{abstract}

Keywords: Physical activity, Screen time, Sleep, Body mass index, Waist circumference, 24-h guidelines, Children

\footnotetext{
* Correspondence: marja.leppanen@folkhalsan.fi

${ }^{1}$ Folkhälsan Research Center, Helsinki, Finland

${ }^{2}$ Department of Food and Nutrition, University of Helsinki, Helsinki, Finland

Full list of author information is available at the end of the article
}

(c) The Author(s). 2019 Open Access This article is distributed under the terms of the Creative Commons Attribution 4.0 International License (http://creativecommons.org/licenses/by/4.0/), which permits unrestricted use, distribution, and reproduction in any medium, provided you give appropriate credit to the original author(s) and the source, provide a link to the Creative Commons license, and indicate if changes were made. The Creative Commons Public Domain Dedication waiver (http://creativecommons.org/publicdomain/zero/1.0/) applies to the data made available in this article, unless otherwise stated. 


\section{Background}

Traditionally, research has focused on investigating associations of physical activity (PA), sedentary time, and sleep with different health outcomes separately, showing evidence of health benefits [1-3]. However, because more recent studies have revealed that these movement behaviors may interact with each other $[4,5]$, there has been growing interest towards an integrated approach to movement behavior. Considering these movement behaviors, PA, sedentary time, and sleep, there were 24-h movement guidelines established for children under 5 years $[6,7]$. In accordance with the guidelines, a healthy 24-h day should include: 1 ) at least $180 \mathrm{~min}$ of total PA (TPA), of which at least $60 \mathrm{~min}$ is moderate-to-vigorous intensity (MVPA), 2) no more than $1 \mathrm{~h}$ of screen time (ST), and 3) 10-13 h of sleep [6]. Previous studies in Canada [8], Australia [9], and Belgium [10] have reported that only $5.6-14.9 \%$ of preschool children adhered to the 24-h movement guidelines. The majority of children complied with the PA and sleep guidelines in Canada and Australia [8, 9], whereas the highest rates in Belgium were meeting the guidelines in sleep and ST [10]. Methodological differences, for instance in assessing PA, explain partly the somewhat different results. Thus, there is a need to confirm the results in different study populations.

To date, the associations of complying with the 24-h movement guidelines and health outcomes in preschool children have been studied little. The Australian research group [9] reported that meeting all three of the 24-h movement guidelines was associated with a better performance in social cognition testing. The Canadian research group [8], however, found no association between compliance of the 24-h movement guidelines with body mass index (BMI). Because obesity is currently a major health concern worldwide [11], it is essential to further study associations between compliance with 24-h movement behaviour and adiposity using multiple adiposity indicators. Furthermore, there are no previous studies investigating the associations in European preschool children.

With the release of the 24-h movement guidelines for the early years $[6,7]$, there is a need to investigate the proportion of children meeting the guidelines and whether the compliance is associated with health outcomes. Such knowledge would help to target health promotion actions to the children and their families that need it the most. The aims of our study were to examine: 1) compliance with the individual and combined 24$\mathrm{h}$ movement guidelines and 2) associations between compliance with both BMI and waist circumference (WC) in Finnish preschool children.

\section{Methods}

\section{Participants and study design}

The present study utilizes data from the DAGIS study (the Increased Health and Wellbeing in Preschools) that aimed to diminish socioeconomic differences in preschool children's energy balance-related behaviors [12]. The study was conducted in early childhood education and care centers (ECEC) in southern and western Finland in 2015-2016. The eligibility criteria for the study were: 1) having at least one group consisting of $3-$ 6-year-old children, 2) providing early education only during the daytime, 3) being Finnish or Swedish speaking (official languages of Finland), and 4) charging income-dependent fees. In total, 864 children (25\% of the invited children) and their families from 66 ECECs (43\% of the invited ECECs) in 8 municipalities agreed to participate in the study. In the present study, complete data on PA, ST, and sleep in overall (including $\geq 3$ weekdays and $\geq 1$ weekend days), weekdays (including $\geq 3$ weekdays), or weekend days (including $\geq 1$ weekend days) were available for 399 boys and 379 girls. Guardians gave their written informed consent. The study was approved by the University of Helsinki Ethical Review Board in the Humanities and Social and Behavioral Sciences in February 2015 (\#6/2015).

\section{Physical activity}

PA was measured using a waist-worn ActiGraph wGT3X-BT accelerometer (Pensacola, FL, USA) for 7 days, $24 \mathrm{~h}$ per day. Parents reported in a diary the nonwearing hours of the accelerometers (e.g., during waterbased activities). A 15-s epoch length was used, and periods of $\geq 10 \mathrm{~min}$ of consecutive zeros were regarded as non-wearing time [13]. A valid day was defined as $\geq 600$ min of awake wearing time. The time spent in different PA intensities was calculated using Butte's cut-points [14]. Overall PA was calculated as: [(mean PA on weekdays $\times 5)+($ mean PA on weekend days $\times 2)] / 7$. To calculate the proportion of children complying with the PA guideline separately for overall, weekdays, and weekend days, PA was dichotomized into $0(<180 \mathrm{~min} /$ day of TPA, of which at least 60 min was MVPA) and $1(\geq 180$ min/day of TPA, of which at least 60 min was MVPA).

\section{Screen time}

Guardians reported ST in a 7-day sedentary behavior diary. The diary was based on a previously validated diary [15], and it was further translated and modified into the Finnish context. Parents were asked to assess the frequency and time (hours/minutes) that their child spent each day: (1) watching TV, (2) watching DVDs or videos, (3) using tablets or smartphones, and (4) using computers or playing computer games. ST is a composition variable of all of the above-mentioned types of activities. Overall ST was calculated as follows: [(mean ST on weekdays $\times 5)+($ mean ST on weekend days $\times 2)] / 7$. To calculate the proportion of children complying with the ST guideline separately for overall, weekdays, and 
weekend days, ST was dichotomized into 0 ( $>60 \mathrm{~min}$ of $\mathrm{ST} /$ day) and 1 ( $\leq 60 \mathrm{~min}$ of ST/day).

\section{Sleep}

Using the same 7-day sedentary behavior diary, guardians were asked to report their child's bedtime and wake-up time. Sleep duration was calculated by subtracting bedtime from wake-up time. Overall sleep duration was calculated as follows: [(mean sleep duration on weekdays $\times 5)+($ mean sleep duration on weekend days $\times$ 2)] / 7. To calculate the proportion of children complying with the sleep guideline separately for overall, weekdays, and weekend days, sleep was dichotomized into 0 (<10 or $>13 \mathrm{~h}$ of sleep/day) and 1 (10-13 h of sleep/ day).

\section{Anthropometry}

Weight, height, and WC were measured by trained researchers. Body weight was measured to the nearest $0.01 \mathrm{~kg}$ with portable bench scales (CAS PB-100/200). Body height was measured to the nearest $0.1 \mathrm{~cm}$ with stadiometers (SECA 217). BMI was calculated as body weight $(\mathrm{kg}) /$ height $^{2}(\mathrm{~m})$, and further, BMI standard deviation score (BMI-SDS) was computed by the national references [16]. Being overweight was defined using the age- and gender-specific BMI cut-offs of the International Obesity Task Force criteria [17]. WC was measured over one layer of clothing twice to the nearest 0.1 $\mathrm{cm}$ with measuring tapes (SECA 201), and the mean of these values was calculated. Waist was defined as the midpoint between the top of the iliac crest and the lower margin of the last palpable rib.

\section{Statistical analysis}

Descriptive information is given as arithmetic means and standard deviations (SD) or frequencies and percentages (\%). Children were categorized as meeting or not meeting: 1) individual guidelines, 2) combinations of any two guidelines, or 3) all three guidelines. Using multiple linear regression, associations with BMI and WC were assessed for: 1) individual guidelines, 2) combinations of any two guidelines, 3) all three guidelines, and 4) the number of guidelines met. Each model was adjusted for the child's age and gender (girl/boy), season of conducting the research (categorized as September-October, November-December, or January-April), and the highest education level in the household [categorized as $<$ bachelor's degree (i.e., comprehensive, vocation, or high school), bachelor's degree (i.e., bachelor's degree or college), or > bachelor's degree (i.e., master's degree or licentiate/doctor)]. The models, including PA, were additionally adjusted for awake wearing time of the accelerometer, and the models, including WC were additionally adjusted for the child's height.
All statistical tests were conducted using a two-sided $5 \%$ level of significance and performed using SPSS Statistics 25 (IBM, Armonk, NY, USA).

\section{Results}

Out of the 864 participating children, 778 (91.2\%) had valid PA, ST, and sleep data in at least one of the three periods (i.e., overall, weekdays, or weekend days). Of those children 721 (92.7\%) had valid data overall, 727 (93.4\%) on weekdays, and 759 (97.6\%) on weekend days.

Table 1 describes the characteristics of the 778 children subdivided by gender. Children's TPA on average was 391 $( \pm 46.2) \mathrm{min} /$ day and they spent $86( \pm 25.5) \mathrm{min} /$ day in MVPA. Furthermore, they spent on average $76( \pm 37.4)$ $\mathrm{min} /$ day on ST and had 10:21 ( $\pm 0: 33)$ h:min/day of sleep. Boys were taller, heavier, had a higher BMI, and spent more time in TPA and MVPA compared to girls $(p<0.05)$.

A total of $23.6 \%$ of children complied with the 24-h movement guidelines overall (Fig. 1a), while the proportion was on weekdays $33.0 \%$ (Fig. 1b) and on weekend days $15.5 \%$ (Fig. 1c). The highest proportion of children met the PA guideline on weekdays $(85.1 \%)$, while the proportion was slightly lower overall (84.6\%) and on weekend days $(74.7 \%)$. The highest proportion of children met the sleep guideline on weekend days (80.9\%), followed by overall (75.7\%) and on weekdays (71.1\%). The compliance with meeting the guideline for ST was the highest on weekdays (52.4\%), followed by overall (35.4\%) and on weekend days (24.5\%). No significant differences were found between boys and girls in terms of meeting the guidelines (data not shown).

Children who met the guidelines for PA overall, on weekdays, or on weekend days had a lower WC compared to children who did not meet the guidelines (Table 2). Moreover, children who met the guidelines for sleep overall and on weekend days had lower BMI and WC compared to children who did not meet the guidelines. Children who met the guidelines for both PA and sleep overall had lower BMI and WC, whereas meeting both guidelines on weekdays or on weekend days was associated with lower WC. There were no significant associations found between meeting all three guidelines (PA, ST, and sleep) and BMI or WC.

Table 3 presents the means of BMI and WC [95\% confidence intervals (CIs)] stratified by the number of participants meeting the guidelines. Meeting two or three guidelines overall compared to zero or one was associated with lower BMI and WC. There were no significant associations found between the number of guidelines met on weekdays or weekend days.

\section{Discussion}

The aims of the present study were to investigate the proportion of Finnish preschool children complying with the 
Table 1 Descriptive characteristics of children

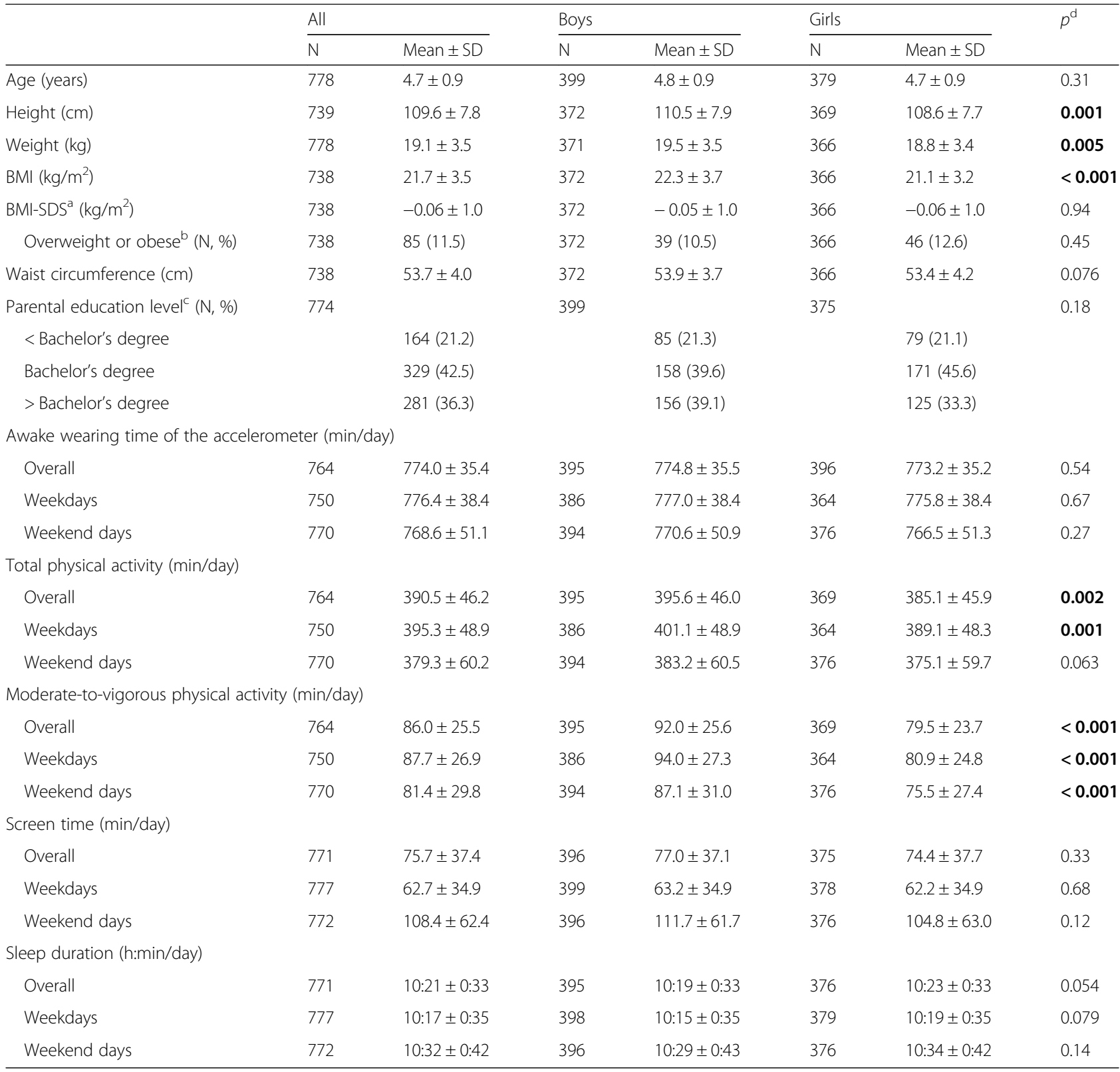

Abbreviations: BMI Body mass index, BMI-SDS Body mass index standard deviation score, SD Standard deviation

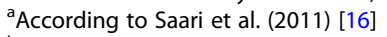

${ }^{\mathrm{b}}$ According to Cole et al. (2012) [17]

'Less than bachelor's degree included comprehensive, vocation, or high school; bachelor's degree included bachelor's degree or college; and more than bachelor's degree included master's degree or licentiate/doctor

${ }^{d}$ T-test for continuous variables and chi-square test for categorized variables. Differences with $P<0.05$ were considered statistically significant (statistically significant values are bolded)

individual (i.e., PA, ST, and sleep) and combined 24-h movement guidelines for the early years as well as the association between complying with the guidelines and anthropometry. In accordance with the results, one-fourth of the preschool children met all three guidelines. Furthermore, meeting guidelines for PA and sleep were associated with lower BMI and WC, while there was no significant association observed regarding ST.
The proportion of children meeting all three guidelines within the 24-h movement guidelines was the highest on weekdays and the lowest on weekend days. This can partly be explained by the somewhat low compliance with the ST guideline. The majority of children met the guideline for PA overall and on weekdays, but on weekend days the highest compliance rate was for the sleep guideline. It is possible that parents might not have the 


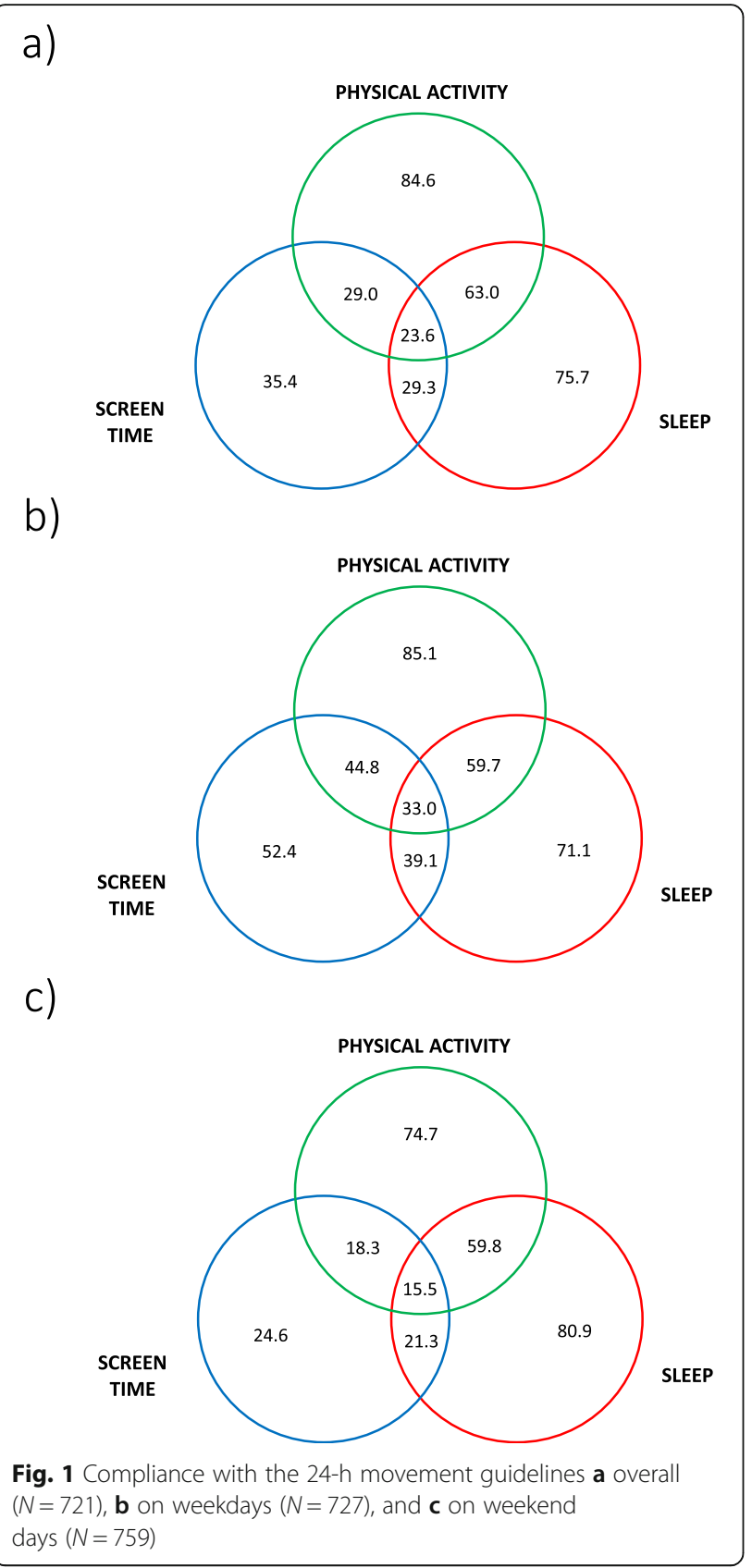

same structure and routines to activate their children at home on weekends than the ECEC's personnel do on weekdays. However, the results highlight the need to increase compliance, especially with ST. In addition, there should be more efforts put on promoting PA, particularly on weekend days, and sleep on weekdays.

There are only three previous studies that have been examined compliance with the 24-h movement guidelines for the early years in preschool children: one in Canada [8], one in Australia [9], and one in Belgium [10]. Similar to our study, the Canadian and Australian studies also reported the highest compliance rates for the PA and sleep guidelines, while the ST guideline received the lowest rates $[8,9]$. In the Belgian study, however, the highest compliance was found for sleep and ST, while it was the lowest for PA [10]. The somewhat different findings can be partly explained by the methodological differences. For instance, in assessing PA the use of a different type of accelerometer device (ActiGraph vs. Actical), the use of different amounts of axes in capturing children's acceleration (triaxial vs. uniaxial), and the use of a different cut-points in defining PA (Butte vs. Pate vs. Evenson) [8-10] can all affect the detected PA. This may further influence the proportion of children meeting the PA guideline. In addition, there were differences in assessing ST and sleep duration (open-ended questions vs. ready-given response categories), which may have affected the results. Due to these differences in methodology and study designs, comparing results to previous studies needs to be done with caution.

To the best of our knowledge, this is the first study that examines the associations of compliance with the 24-h movement guidelines for the early years with anthropometry in European preschool children. In accordance with our results, meeting the guidelines separately for PA or sleep, or for both of them, was associated with lower WC, whereas meeting the guidelines for sleep, or both PA and sleep, was associated with lower BMI. The Canadian study previously reported no significant associations between compliance with the 24-h movement guidelines and BMI in preschoolers [8], yet the differences in study designs need to be kept in mind. However, our results are in line with a large 12-country study in 9-11-year-old children $(N=6128)$ that investigated associations of compliance with the 24-h movement guidelines with BMI [18]. Although their guidelines were slightly different to the ones in younger children, they found that meeting all three guidelines regarding PA, ST, and sleep was associated with lower BMI. Moreover, we found that meeting two or three guidelines instead of none or one was associated with lower BMI and WC. These findings support the theory that these three movement behaviors are co-dependent, and thus, there is a great need for interventions that attempt to promote compliance of more than one movement behavior at a time.

Our results showed that the associations of meeting the guidelines for PA and/or sleep appeared to be stronger with WC compared to BMI. As an elevated BMI has been suggested to indicate more "overweight" than "over fatness" [19], WC has been commonly used as a marker for central obesity [20]. Thus, the findings indicate that meeting the guidelines for PA and sleep may have a role in decreasing visceral adipose tissue, which in turn has been related to adverse metabolic health consequences [20]. However, although we used accurate measures of anthropometry, we were not able to address associations 
Table 2 Associations of meeting the recommendations for the 24-h movement guidelines with BMl and waist circumference

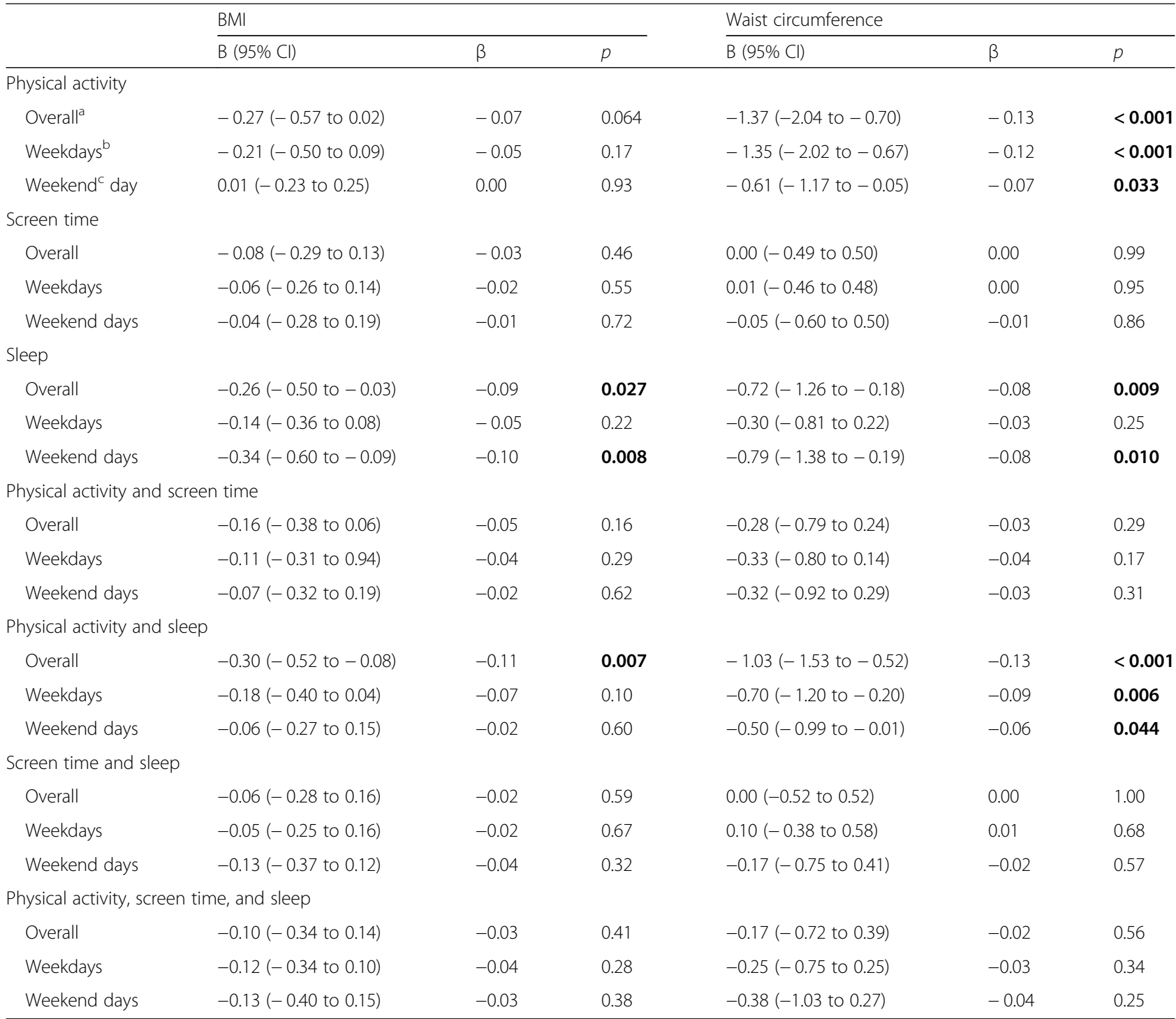

Values are unstandardized (B) and standardized regression coefficients $(\beta)$ with their $p$-values from linear regression analyses (statistically significant values are bolded). All models were adjusted for age, gender, season, and socioeconomic status (the highest education level in the family). Models including PA were additionally adjusted for awake wearing time of accelerometer, whereas models including waist circumference were additionally adjusted for height Abbreviations: $B M I$ Body mass index

${ }^{\mathrm{a}} N=694,{ }^{\mathrm{b}} \mathrm{N}=700,{ }^{\mathrm{c}} \mathrm{N}=715$

between compliance with 24-h movement guidelines and body composition, such as fat mass or fat-free mass. Thus, in future studies, the use of more detailed measurements of body composition should be considered.

We found no significant association between meeting the guideline for ST with BMI or WC. It is possible that the rather short time, $\leq 60 \mathrm{~min} /$ day, was not enough to show the difference. Although ST has been connected to adverse health consequences, such as depressive symptoms and a poorer quality of health in children [21], our finding is encouraging. The use of ST has also been considered a way to promote children's digital technology skills as well as to enhance learning and creating competence in social interaction [22]. However, it is essential to further examine the associations between ST and different health outcomes, and to clarify, for instance, the role of ST duration in order to support children's health.

\section{Strengths and limitations}

The strengths of the present study include a relatively large sample of children as well as comprehensive assessments of PA, ST, and sleep duration. We used triaxial accelerometers instead of uniaxial in order to take 
Table 3 Associations of the number of guidelines met with BMI and waist circumference

\begin{tabular}{|c|c|c|c|c|c|c|}
\hline & \multicolumn{3}{|l|}{ Mean $(95 \% \mathrm{Cl})$} & \multicolumn{3}{|c|}{ Mean $(95 \% \mathrm{Cl})$ difference between groups } \\
\hline & $\begin{array}{l}\text { Meeting } 0^{a} \text { or } 1^{b} \\
\text { guideline }\end{array}$ & $\begin{array}{l}\text { Meeting } 2^{c} \\
\text { guidelines }\end{array}$ & $\begin{array}{l}\text { Meeting } 3^{d} \\
\text { guidelines }\end{array}$ & $\overline{2-1}$ & $3-1$ & $3-2$ \\
\hline \multicolumn{7}{|l|}{ Overall } \\
\hline BMl & 16.1 (15.9 to 16.3$)$ & $\begin{array}{l}15.7(15.6 \text { to } \\
15.8)\end{array}$ & $\begin{array}{l}15.7(15.5 \text { to } \\
15.9)\end{array}$ & $\begin{array}{l}-0.39(-0.63 \text { to }-0.15) \\
\boldsymbol{p}=\mathbf{0 . 0 0 2}\end{array}$ & $\begin{array}{l}-0.38(-0.66 \text { to }-0.10) \\
\boldsymbol{p}=\mathbf{0 . 0 0 8}\end{array}$ & $\begin{array}{l}0.01(-0.24 \text { to } 0.26) \\
p=0.94\end{array}$ \\
\hline $\begin{array}{l}\text { Waist } \\
\text { circumference }\end{array}$ & 54.5 (53.7 to 55.2$)$ & $\begin{array}{l}53.2(52.8 \text { to } \\
53.6)\end{array}$ & $\begin{array}{l}53.5(53.0 \text { to } \\
54.0)\end{array}$ & $\begin{array}{l}-1.19(-1.73 \text { to }-0.63) \\
\boldsymbol{p}<\mathbf{0 . 0 0 1}\end{array}$ & $\begin{array}{l}-1.73(-1.68 \text { to }-0.38) \\
\boldsymbol{p}=\mathbf{0 . 0 0 2}\end{array}$ & $\begin{array}{l}0.16(-0.41 \text { to } 0.73) \\
p=0.58\end{array}$ \\
\hline \multicolumn{7}{|l|}{ Weekdays } \\
\hline BMI & 15.9 (15.7 to 16.2$)$ & $\begin{array}{l}15.8(15.7 \text { to } \\
16.0)\end{array}$ & $\begin{array}{l}15.7(15.6 \text { to } \\
15.9)\end{array}$ & $\begin{array}{l}-0.11(-0.37 \text { to } 0.15) \\
p=0.42\end{array}$ & $\begin{array}{l}-0.20(-0.47 \text { to } 0.08) \\
p=0.16\end{array}$ & $\begin{array}{l}-0.09(-0.32 \text { to } 0.15), \\
p=0.46\end{array}$ \\
\hline $\begin{array}{l}\text { Waist } \\
\text { circumference }\end{array}$ & $54.0(53.2$ to 54.7$)$ & $\begin{array}{l}53.5(53.0 \text { to } \\
53.9)\end{array}$ & $\begin{array}{l}53.6(53.3 \text { to } \\
54.0)\end{array}$ & $\begin{array}{l}-0.53(-1.13 \text { to } 0.07), \\
p=0.082\end{array}$ & $\begin{array}{l}-0.63(-1.26 \text { to } 0.00), \\
p=0.05\end{array}$ & $\begin{array}{l}-0.10(-0.63 \text { to } 0.44), \\
p=0.72\end{array}$ \\
\hline \multicolumn{7}{|l|}{ Weekend days } \\
\hline BMI & 15.9 (15.7 to 16.0$)$ & $\begin{array}{l}15.8(15.7 \text { to } \\
16.0)\end{array}$ & $\begin{array}{l}15.7(15.5 \text { to } \\
15.9)\end{array}$ & $\begin{array}{l}-0.02(-0.24 \text { to } 0.21) \\
p=0.89\end{array}$ & $\begin{array}{l}-0.17(-0.47 \text { to } 0.14) \\
p=0.29\end{array}$ & $\begin{array}{l}-0.15(-0.44 \text { to } 0.14), \\
p=0.31\end{array}$ \\
\hline $\begin{array}{l}\text { Waist } \\
\text { circumference }\end{array}$ & 53.9 (53.3 to 54.5$)$ & $\begin{array}{l}53.7(53.3 \text { to } \\
54.1)\end{array}$ & $\begin{array}{l}53.0(52.4 \text { to } \\
53.6)\end{array}$ & $\begin{array}{l}-0.35(-0.88 \text { to } 0.18) \\
p=0.19\end{array}$ & $\begin{array}{l}-0.67(-1.39 \text { to } 0.05) \\
p=0.069\end{array}$ & $\begin{array}{l}-0.32(-0.99 \text { to } 0.36), \\
p=0.36\end{array}$ \\
\hline
\end{tabular}

Linear regression models were adjusted for age, gender, season, and socioeconomic status (statistically significant values are bolded)

Abbreviations: BMI Body mass index, $\mathrm{Cl}$ Confidence interval

a Overall $N=14$, Weekdays $N=14$, Weekend days $N=28$

boverall $N=173$, Weekdays $N=149$, Weekend days $N=212$

'Overall $N=364$, Weekdays $N=324$, Weekend days $N=401$

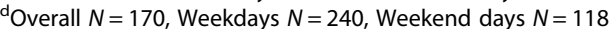

into account children's intermittent patterns of movement [13]. In addition, we chose to use the same cutpoints that have also been used in other recent European studies on preschool children, increasing the comparability between the studies [23, 24]. The use of daily ST and sleep diaries with open questions instead of readygiven response categories in assessing ST and sleep were chosen to increase representativeness of ST and sleep. Finally, the daily ST diary included all types of ST (i.e., TV viewing, watching DVDs or videos, using tablets or smartphones, and using computers or playing computer games) instead of restricting it only to TV viewing [21].

The study also has some limitations that need to be considered. Firstly, the cross-sectional study design limits the conclusion about causality between the observed associations. Secondly, the sleep diary was filled in by the parents, and therefore, we have no information about whether the children had a nap while in ECECs. Thus, it is possible that the actual amount of sleep per day was slightly longer, which could increase the proportion of children meeting the guideline for sleep. Thirdly, we did not include any dietary factors (children's energy intake) as we aimed to provide results that were well comparable with those of Chaput et al. [8]. Finally, due to the relatively low participation rate, the sample may be somewhat selected with participants interested in healthy lifestyles. This may have led to higher compliance rates than in the general population.

\section{Conclusions}

Only one-fourth of the children met all three guidelines of the 24-h movement guidelines for the early years. The majority of preschool children met the guidelines for PA, followed by sleep. The lowest compliance was found for ST. Meeting recommendations for both PA and sleep was associated with a healthier weight status. However, there is still a need to improve compliance with the 24-h movement guidelines, especially for ST, in order to support health in young children.

\section{Abbreviations}

BMI: Body mass index; BMI-SDS: Body mass index standard deviation score; Cl: Confidence interval; ECEC: Early childhood education and care center; MVPA: Moderate to vigorous physical activity; PA: Physical activity; SD: Standard deviation; ST: Screen time; TPA: Total physical activity; WC: Waist circumference

\section{Acknowledgements \\ The authors thank the participating families, the early childhood education and care centers, and the members of the DAGIS research group for their help regarding recruitment and data collection. The preliminary results of this study have been previously published in the ISBNPA Advancing Behaviour Change Science abstract book: \\ https://www.isbnpa.org/files/articles/2019/06/25/122/attachments/5d124e1 face47.pdf.}

\section{Authors' contributions}

ER is the principal investigator for the DAGIS study and designed this research together with all coauthors. MHL was responsible for data analysis and drafted the manuscript, which was subsequently reviewed by $C R$, HW, $C A, K S, L K, M E$, and ER All authors approved the final version. 


\section{Funding}

This research was funded by Folkhälsan Research Center, University of Helsinki, The Ministry of Education and Culture in Finland, The Ministry of Social Affairs and Health, The Academy of Finland (Grants: 285439, 287288, 288038), The Juho Vainio Foundation, The Signe and Ane Gyllenberg Foundation, The Finnish Cultural Foundation/South Ostrobothnia Regional Fund, The Päivikki and Sakari Sohlberg Foundation, Medicinska Föreningen Liv och Hälsa, Finnish Foundation for Nutrition Research, and Finnish Food Research Foundation. None of the funding bodies had a role in designing the study, collecting, analyzing, or interpreting the data, or writing the manuscript.

\section{Availability of data and materials}

Not applicable.

\section{Ethics approval and consent to participate}

The study was approved by the University of Helsinki Ethical Review Board in the Humanities and Social and Behavioral Sciences in February 2015 (\#6/ 2015). Guardians gave their written informed consent.

\section{Consent for publication}

Not applicable.

\section{Competing interests}

The authors declare that they have no competing interests.

\section{Author details}

${ }^{1}$ Folkhälsan Research Center, Helsinki, Finland. ${ }^{2}$ Department of Food and Nutrition, University of Helsinki, Helsinki, Finland. ${ }^{3}$ Finnish Institute for Health and Welfare, Helsinki, Finland. ${ }^{4}$ Department of Biosciences and Nutrition, Karolinska Institutet, Huddinge, Sweden. ${ }^{5}$ Faculty of Educational Sciences, University of Helsinki, Helsinki, Finland. ${ }^{6}$ Department of Social Research, University of Turku, Turku, Finland. 'Department of Public Health, Clinicum, University of Helsinki, Helsinki, Finland.

Received: 16 August 2019 Accepted: 19 November 2019

Published online: 03 December 2019

\section{References}

1. Carson V, Lee E, Hewitt L, Jennings C, Hunter S, Kuzik N, et al. Systematic review of the relationships between physical activity and health indicators in the early years (0-4 years). BMC Public Health. 2017;17(Suppl 5):854-63.

2. Poitras VJ, Gray CE, Janssen X, Aubert S, Carson V, Faulkner G, et al. Systematic review of the relationships between sedentary behaviour and health indicators in the early years (0-4 years). BMC Public Health. 2017 : 17(Suppl 5):868-89.

3. Chaput J, Gray CE, Poitras VJ, Carson V, Gruber R, Birken CS, et al. Systematic review of the relationships between sleep duration and health indicators in the early years (0-4 years). BMC Public Health. 2017;17(Suppl 5):855-107.

4. Chastin S, Palarea-Albaladejo J, Dontje ML, Skelton DA. Combined effects of time spent in physical activity, sedentary behaviors and sleep on obesity and cardio-metabolic health markers: a novel compositional data analysis approach. PLoS One. 2015;10(10):e0139984.

5. Chaput J, Saunders TJ, Carson V. Interactions between sleep, movement and other non-movement behaviours in the pathogenesis of childhood obesity. Obes Rev. 2017;18:7-14.

6. Tremblay MS, Chaput J, Adamo KB, Aubert S, Barnes JD, Choquette L, et al Canadian 24-hour movement guidelines for the early years ( $0-4$ years): an integration of physical activity, sedentary behaviour, and sleep. BMC Public Health. 2017:17(Suppl 5):874-32.

7. Okely AD, Ghersi D, Hesketh KD, Santos R, Loughran SP, Cliff DP, et al. A collaborative approach to adopting/adapting guidelines - The Australian 24Hour Movement Guidelines for the early years (Birth to 5 years): an integration of physical activity, sedentary behavior, and sleep. BMC Public Health. 2017;17(Suppl 5):869-190.

8. Chaput J, Colley RC, Aubert S, Carson V, Janssen I, Roberts KC, et al. Proportion of preschool-aged children meeting the Canadian 24-Hour Movement Guidelines and associations with adiposity: results from the Canadian Health Measures Survey. BMC Public Health. 2017;17(Suppl 5):829154.
9. Cliff DP, McNeill J, Vella SA, Howard SJ, Santos R, Batterham M, et al. Adherence to 24-Hour Movement Guidelines for the Early Years and associations with social-cognitive development among Australian preschool children. BMC Public Health. 2017;17(Suppl 5):857-215.

10. De Craemer M, McGregor D, Androutsos O, Manios Y, Cardon G. Compliance with 24-h Movement Behaviour Guidelines among Belgian PreSchool Children: The ToyBox-Study. Int J Environ Res Public Health. 2018; 15(10):2171.

11. Ng M, Fleming T, Robinson M. Global, regional, and national prevalence of overweight and obesity in children and adults during 1980-2013: a systematic analysis for the global burden of disease study 2013. Lancet. 2014;384(9945):766-81.

12. Lehto E, Ray C, Vepsäläinen H, Korkalo L, Lehto R, Kaukonen R, et al. Increased Health and Wellbeing in Preschools (DAGIS) Study_Differences in Children's Energy Balance-Related Behaviors (EBRBs) and in Long-Term Stress by Parental Educational Level. Int J Environ Res Public Health. 2018; 15(10):2313.

13. Cliff DP, Reilly JJ, Okely AD. Methodological considerations in using accelerometers to assess habitual physical activity in children aged 0-5 years. J Sci Med Sport. 2008;12(5):557-67.

14. Butte NF, Wong WW, Lee JS, Adolph AL, Puyau MR, Zakeri IF. Prediction of energy expenditure and physical activity in preschoolers. Med Sci Sports Exerc. 2014 Jun;46(6):1216-26.

15. Wen LM, van der Ploeg HP, Kite J, Cashmore A, Rissel C. A validation study of assessing physical activity and sedentary behavior in children aged 3 to 5 years. Pediatr Exerc Sci. 2010;22(3):408-20.

16. Saari A, Sankilampi U, Hannila M, Kiviniemi V, Kesseli K, Dunkel L. New Finnish growth references for children and adolescents aged 0 to 20 years: length/height-for-age, weight-for-length/height, and body mass index-forage. Ann Med. 2011;43(3):235-48.

17. Cole TJ, Lobstein T. Extended international (IOTF) body mass index cut-offs for thinness, overweight and obesity. Pediatr Obes. 2012;7(4):284-94.

18. Roman-Viñas B, Chaput J, Katzmarzyk PT, Fogelholm M, Lambert EV, Maher C, et al. Proportion of children meeting recommendations for 24-hour movement guidelines and associations with adiposity in a 12-country study. Int J Behav Nutr Phys Act. 2016;13(1):123.

19. Rolland-Cachera MF. Childhood obesity: current definitions and recommendations for their use. Int J Pediatr Obes. 2011 Oct;6(5-6):325-31.

20. Bays H. Central obesity as a clinical marker of adiposopathy; increased visceral adiposity as a surrogate marker for global fat dysfunction. Curr Opin Endocrinol Diabetes Obes. 2014;21(5):345-51.

21. Stiglic N, Viner RM. Effects of screentime on the health and well-being of children and adolescents: a systematic review of reviews. BMJ Open. 2019; 9(1):e023191.

22. Straker L, Zabatiero J, Danby S, Thorpe K, Edwards S. Conflicting guidelines on young Children's screen time and use of digital technology create policy and practice dilemmas. J Pediatr. 2018;202:300-3.

23. Berglind D, Ljung R, Tynelius P, Brooke HL. Cross-sectional and prospective associations of meeting 24-h movement guidelines with overweight and obesity in preschool children. Pediatr Obes. 2018;13(7):442-9.

24. Arhab A, Messerli-Bürgy N, Kakebeeke TH, Lanzi S, Stülb K, Zysset AE, et al. Childcare correlates of physical activity, sedentary behavior, and adiposity in preschool children: a cross-sectional analysis of the SPLASHY study. J Environ Public Health. 2018;2018:9157194-12.

\section{Publisher's Note}

Springer Nature remains neutral with regard to jurisdictional claims in published maps and institutional affiliations. 